\title{
QUASI BOUNDARY TRIPLES, SELF-ADJOINT EXTENSIONS, AND ROBIN LAPLACIANS ON THE HALF-SPACE
}

\author{
JUSSI BEHRNDT AND PETER SCHLOSSER
}

\begin{abstract}
In this note self-adjoint extensions of symmetric operators are investigated by using the abstract technique of quasi boundary triples and their Weyl functions. The main result is an extension of [5 Theorem 2.6] which provides sufficient conditions on the parameter in the boundary space to induce self-adjoint realizations. As an example self-adjoint Robin Laplacians on the half-space with boundary conditions involving an unbounded coefficient are considered.
\end{abstract}

\section{INTRODUCTION}

The concept of quasi boundary triples and their Weyl functions is a useful tool in the spectral theory of symmetric and self-adjoint elliptic partial differential operators. This abstract notion from [3, 4 is a slight generalization of ordinary boundary triples and their Weyl functions from 11, 14, 19, adapted and extended in such a way that it directly applies to elliptic boundary value problems in the Hilbert space framework.

Very roughly speaking, a quasi boundary triple consists of a boundary Hilbert space $\mathcal{G}$ - in applications typically the $L^{2}$-space on the boundary of some domain $\Omega$ - and two boundary mappings $\Gamma_{0}$ and $\Gamma_{1}$ that satisfy an abstract second Green identity. A natural choice are the Neumann and Dirichlet trace operators if one deals with the Laplacian in $L^{2}(\Omega)$. The boundary mappings are defined on the domain of some operator $T$ which is a core of the maximal operator; in the case of the Laplacian, the core $H^{2}(\Omega)$ is often a convenient choice. The Weyl function corresponding to a quasi boundary triple can be viewed as the abstract counterpart of the Dirichlet-to-Neumann map and is an important analytic object since it can be used to characterize the spectrum of the self-adjoint realizations in this theory. One uses abstract boundary conditions to define restrictions of $T$ in the form

$$
A_{[B]} f=T f, \quad \operatorname{dom} A_{[B]}=\left\{f \in \operatorname{dom} T: \Gamma_{0} f=B \Gamma_{1} f\right\},
$$

where $B$ is an operator in the boundary space $\mathcal{G}$. It is an immediate consequence of the abstract second Green identity that a symmetric operator $B$ leads to a symmetric operator $A_{[B]}$, but in general a self-adjoint boundary parameter $B$ does not induce a self-adjoint operator $A_{[B]}$ - a fact that is not too surprising when taking into account that the range of the boundary mappings is not necessarily the whole boundary space $\mathcal{G}$; cf. Definition 2.1 .

It is one of the main objectives of the present note to provide a new useful sufficient condition on the boundary parameter $B$ and the properties of the Weyl function to ensure self-adjointness of the extension $A_{[B]}$. Here we generalize a recent result from 5 by allowing boundary operators $B$ that are factorized in the form $B=B_{1} B_{2}$, or more general $B \subset B_{1} B_{2}$. The assumptions on $B$ in [5, Theorem 2.6] 
are here replaced by similar ones on $B_{1}$ and $B_{2}$. We refer the reader to Theorem 2.2 and the discussion afterwards for more details.

As an example and illustration for the abstract techniques we discuss the Laplacian on the half-space $\mathbb{R}_{+}^{d}=\left\{x \in \mathbb{R}^{d}: x_{d}>0\right\}$ in any dimension $d \geq 2$ in Section 3 . The key feature is that Theorem 2.2 and Corollary 2.3 can be applied for the Laplace operator with Robin boundary conditions $\tau_{N} f=\alpha \tau_{D} f$ on $\partial \mathbb{R}_{+}^{d} \simeq \mathbb{R}^{d-1}$, where

$$
\alpha \in L^{p}\left(\mathbb{R}^{d-1}\right)+L^{\infty}\left(\mathbb{R}^{d-1}\right)
$$

is real-valued. In Theorem 3.5 we have the slightly stronger assumption $p>\frac{4}{3}(d-1)$ if $d \geq 3$ and $p>2$ if $d=2$ than the usual form method requires (namely, $p=$ $d-1$ if $d \geq 3$ and $p>1$ if $d=2$ is sufficient by Proposition 3.8), but also at the same time a higher Sobolev regularity for the operator domain. For other related variants of Theorem 3.5 we also refer the reader to [1, Theorem 7.2] which provides $H^{2}$-regularity for more general second order elliptic differential expressions on certain unbounded non-smooth domains (see Remark 3.7), to [18, Theorem 4.5 and Lemma 5.3] for the case of Laplacians on bounded Lipschitz domains, and to [17. Section 2]. In this context we also mention the contributions [1, 6, 1, 16, 30, 31] dealing with Robin Laplacians with singular boundary conditions and we refer to [2, 9, 10, 12, 21, 23, 24, 25, 27, 28, 29, 32, 33, 34, for some other recent works on spectral problems for Robin Laplacians.

\section{ACKNOWLEDGEMENTS}

Jussi Behrndt is most grateful to the organizers of the conference Mathematics, Signal Processing and Linear Systems: New Problems and Directions, November 2017, at Chapman University, Orange, California, for creating a stimulating atmosphere and for the great hospitality. The authors also wish to thank Vladimir Lotoreichik, Konstantin Pankrashkin, Nicolas Popoff, and Jonathan Rohleder for helpful comments and remarks. Both authors gratefully acknowledge financial support by the Austrian Science Fund (FWF), project P 25162-N26, and the Austria-Czech Republic cooperation grant CZ02/2017 by the Austrian Agency for International Cooperation in Education and Research (OeAD).

\section{QUASI BOUNDARY TRIPLES AND SELF-ADJOINT EXTENSIONS}

In this section we first recall the notion of quasi boundary triples and their Weyl functions in the extension theory of symmetric operators from [3, 4. Afterwards we provide a new sufficient criterion for self-adjointness in Theorem 2.2, which is the main abstract result in this note.

In the following let $\mathcal{H}$ be a Hilbert space with inner product $(\cdot, \cdot)_{\mathcal{H}}$. The next definition is a generalization of the concept of ordinary and generalized boundary triples; cf. [11, 13, 14, 15, 19].

Definition 2.1. Let $S$ be a densely defined, closed, symmetric operator in $\mathcal{H}$ and let $T$ be a closable operator with $\bar{T}=S^{*}$. A triple $\left\{\mathcal{G}, \Gamma_{0}, \Gamma_{1}\right\}$ is a quasi boundary triple for $T \subset S^{*}$ if $\left(\mathcal{G},(\cdot, \cdot)_{\mathcal{G}}\right)$ is a Hilbert space and the linear mappings $\Gamma_{0}, \Gamma_{1}: \operatorname{dom} T \rightarrow \mathcal{G}$ satisfy the following conditions (i)-(iii).

(i) The abstract second Green identity

$$
(T f, g)_{\mathcal{H}}-(f, T g)_{\mathcal{H}}=\left(\Gamma_{1} f, \Gamma_{0} g\right)_{\mathcal{G}}-\left(\Gamma_{0} f, \Gamma_{1} g\right)_{\mathcal{G}}
$$

holds for all $f, g \in \operatorname{dom} T$. 
(ii) The range of $\left(\Gamma_{0}, \Gamma_{1}\right)^{\top}: \operatorname{dom} T \rightarrow \mathcal{G} \times \mathcal{G}$ is dense.

(iii) The operator $A_{0}:=T \uparrow \operatorname{ker} \Gamma_{0}$ is self-adjoint in $\mathcal{H}$.

Recall from [3, 4] that for a densely defined, closed, symmetric operator $S$ in $\mathcal{H}$ a quasi boundary triple $\left\{\mathcal{G}, \Gamma_{0}, \Gamma_{1}\right\}$ exists if and only if the deficiency indices of $S$ coincide. In this case one has $\operatorname{dom} S=\operatorname{ker} \Gamma_{0} \cap \operatorname{ker} \Gamma_{1}$. The notion of quasi boundary triples reduces to the well-known concept of ordinary boundary triples if $T=S^{*}$. For more details we refer the reader to 3,4 .

Assume now that $\left\{\mathcal{G}, \Gamma_{0}, \Gamma_{1}\right\}$ is a quasi boundary triple for $T \subset S^{*}$. In a similar way as for ordinary and generalized boundary triples in [14, 15] one associates the $\gamma$-field and the Weyl function. Their definition and some of their properties will now be recalled very briefly. Again we refer the reader to 3, 4, for a more detailed exposition. Observe first that the direct sum decomposition

$$
\operatorname{dom} T=\operatorname{dom} A_{0} \dot{+} \operatorname{ker}(T-\lambda)=\operatorname{ker} \Gamma_{0} \dot{+} \operatorname{ker}(T-\lambda), \quad \lambda \in \rho\left(A_{0}\right),
$$

implies that $\Gamma_{0}\left\lceil\operatorname{ker}(T-\lambda)\right.$ is invertible for $\lambda \in \rho\left(A_{0}\right)$. The $\gamma$-field $\gamma$ and Weyl function $M$ are then defined as operator-valued functions on $\rho\left(A_{0}\right)$ by

$$
\lambda \mapsto \gamma(\lambda):=\left(\Gamma_{0}\lceil\operatorname{ker}(T-\lambda))^{-1} \text { and } \lambda \mapsto M(\lambda):=\Gamma_{1} \gamma(\lambda),\right.
$$

respectively. It is clear from (2.2) that $\operatorname{dom} \gamma(\lambda)=\operatorname{dom} M(\lambda)=\operatorname{ran} \Gamma_{0}$ for all $\lambda \in \rho\left(A_{0}\right)$. Moreover, the values $\gamma(\lambda)$ of the $\gamma$-field are densely defined and bounded operators from $\mathcal{G}$ into $\mathcal{H}$ such that $\operatorname{ran} \gamma(\lambda)=\operatorname{ker}(T-\lambda)$. With the help of the abstract second Green identity in (2.1) one verifies the representation

$$
\gamma(\bar{\lambda})^{*}=\Gamma_{1}\left(A_{0}-\lambda\right)^{-1}, \quad \lambda \in \rho\left(A_{0}\right),
$$

of the adjoint $\gamma$-field, which is a bounded and everywhere defined operator from $\mathcal{H}$ into $\mathcal{G}$. The values $M(\lambda)$ of the Weyl function are operators in $\mathcal{G}$ which are not necessarily closed and in general unbounded. Note that also $\operatorname{ran} M(\lambda) \subset \operatorname{ran} \Gamma_{1}$ by definition.

For a given quasi boundary triple $\left\{\mathcal{G}, \Gamma_{0}, \Gamma_{1}\right\}$ and an operator $B$ in $\mathcal{G}$ the extension $A_{[B]}$ of $S$ in $\mathcal{H}$ is defined as

$$
A_{[B]} f=T f, \quad \operatorname{dom} A_{[B]}=\left\{f \in \operatorname{dom} T: \Gamma_{0} f=B \Gamma_{1} f\right\} .
$$

In contrast to ordinary boundary triples (see [11, 14, 19]) a self-adjoint boundary operator $B$ in $\mathcal{G}$ does not necessarily induce a self-adjoint extension $A_{[B]}$ in $\mathcal{H}$. There are various results in the literature that provide sufficient conditions for this conclusion to hold, see, e.g., 3, 4, 5. Our aim in the next theorem is to provide a useful generalization of a recent result in [5]; cf. Corollary 2.4.

Theorem 2.2. Let $S$ be a densely defined, closed, symmetric operator in $\mathcal{H}$ and let $\left\{\mathcal{G}, \Gamma_{0}, \Gamma_{1}\right\}$ be a quasi boundary triple for $T \subset S^{*}$ with $A_{0}=T \uparrow \operatorname{ker} \Gamma_{0}, \gamma$-field $\gamma$ and Weyl function $M$. Let $\lambda_{0} \in \rho\left(A_{0}\right) \cap \mathbb{R}$ and let $B$ be a symmetric operator in $\mathcal{G}$. Assume that $B \subset B_{1} B_{2}$ holds with some operators $B_{1}, B_{2}$ in $\mathcal{G}$ and that the following conditions are satisfied.

(i) $1 \in \rho\left(B_{2} \overline{M\left(\lambda_{0}\right) B_{1}}\right)$;

(ii) $\operatorname{ran}\left(B_{2} \overline{M\left(\lambda_{0}\right) B_{1}}\right) \subset \operatorname{ran} \Gamma_{0} \cap \operatorname{dom} B_{1}$;

(iii) $\operatorname{ran}\left(B_{1}\left\lceil\operatorname{ran} \Gamma_{0}\right) \subset \operatorname{ran} \Gamma_{0}\right.$;

(iv) $\operatorname{ran}\left(B_{2}\left\lceil\operatorname{ran} \Gamma_{1}\right) \subset \operatorname{ran} \Gamma_{0}\right.$;

(v) $\operatorname{ran} \Gamma_{1} \subset \operatorname{dom} B$. 
Then the extension $A_{[B]}$ in (2.5) is a self-adjoint operator in $\mathcal{H}$ and for every $\lambda \in \rho\left(A_{0}\right) \cap \rho\left(A_{[B]}\right)$ the Krein type resolvent formula

$$
\left(A_{[B]}-\lambda\right)^{-1}-\left(A_{0}-\lambda\right)^{-1}=\gamma(\lambda) B_{1}\left(1-B_{2} M(\lambda) B_{1}\right)^{-1} B_{2} \gamma(\bar{\lambda})^{*}
$$

is valid.

In the next corollary the special case that $\operatorname{ran} \Gamma_{0}=\mathcal{G}$ is formulated. In this situation the quasi boundary triple $\left\{\mathcal{G}, \Gamma_{0}, \Gamma_{1}\right\}$ is a generalized boundary triple in the sense of [13, 15. It is clear that condition (ii) in Theorem 2.2 simplifies and that conditions (iii) and (iv) are automatically satisfied in this case.

Corollary 2.3. Let $\left\{\mathcal{G}, \Gamma_{0}, \Gamma_{1}\right\}, A_{0}=T \uparrow \operatorname{ker} \Gamma_{0}, M$ and $\lambda_{0} \in \rho\left(A_{0}\right) \cap \mathbb{R}$, and $B \subset B_{1} B_{2}$ be as in Theorem 2.2. Assume, in addition, that $\operatorname{ran} \Gamma_{0}=\mathcal{G}$ and that the following conditions are satisfied.

(i) $1 \in \rho\left(B_{2} \overline{M\left(\lambda_{0}\right) B_{1}}\right)$;

(ii) $\operatorname{ran}\left(B_{2} \overline{\left.M\left(\lambda_{0}\right) B_{1}\right)} \subset \operatorname{dom} B_{1}\right.$;

(iii) $\operatorname{ran} \Gamma_{1} \subset \operatorname{dom} B$.

Then the extension $A_{[B]}$ in (2.5) is a self-adjoint operator in $\mathcal{H}$ and for every $\lambda \in \rho\left(A_{0}\right) \cap \rho\left(A_{[B]}\right)$ the Krein type resolvent formula (2.6] is valid.

The next corollary shows that for the special choice $B_{1}=I_{\mathcal{G}}$ and $B_{2}=B$ Theorem 2.2 coincides with [5, Theorem 2.6].

Corollary 2.4. Let $\left\{\mathcal{G}, \Gamma_{0}, \Gamma_{1}\right\}, A_{0}=T \uparrow \operatorname{ker} \Gamma_{0}, M, \gamma$ and $\lambda_{0} \in \rho\left(A_{0}\right) \cap \mathbb{R}$ be as in Theorem 2.2 and assume that $B$ is a symmetric operator in $\mathcal{G}$ such that the following conditions are satisfied.

(i) $1 \in \rho\left(B \overline{M\left(\lambda_{0}\right)}\right)$;

(ii) $\operatorname{ran}\left(B \overline{M\left(\lambda_{0}\right)}\right) \subset \operatorname{ran} \Gamma_{0}$;

(iii) $\operatorname{ran}\left(B \nmid \operatorname{ran} \Gamma_{1}\right) \subset \operatorname{ran} \Gamma_{0}$;

(iv) $\operatorname{ran} \Gamma_{1} \subset \operatorname{dom} B$.

Then the extension $A_{[B]}$ in (2.5) is a self-adjoint operator in $\mathcal{H}$ and for every $\lambda \in \rho\left(A_{0}\right) \cap \rho\left(A_{[B]}\right)$ the Krein type resolvent formula

$$
\left(A_{[B]}-\lambda\right)^{-1}-\left(A_{0}-\lambda\right)^{-1}=\gamma(\lambda)(1-B M(\lambda))^{-1} B \gamma(\bar{\lambda})^{*}
$$

is valid.

Remark 2.5. The assumption $\lambda_{0} \in \rho\left(A_{0}\right) \cap \mathbb{R}$ and conditions (i)-(ii) in Theorem 2.2 (and similarly in Corollary 2.3 and Corollary 2.4 ) can be replaced by assuming that there exist $\lambda_{ \pm} \in \mathbb{C}^{ \pm}$with the properties

(i') $1 \in \rho\left(B_{2} \overline{M\left(\lambda_{ \pm}\right) B_{1}}\right)$;

(ii') $\operatorname{ran}\left(B_{2} \overline{M\left(\lambda_{ \pm}\right) B_{1}}\right) \subset \operatorname{ran} \Gamma_{0} \cap \operatorname{dom} B_{1}$.

Proof of Theorem 2.2. The proof is split into four separate steps: First the selfadjointness of $A_{[B]}$ is shown in Steps 1 and 2 and afterwards, in Step 3 and 4, the resolvent formula (2.6) is verified.

Step 1. In this step we prove the inclusion

$$
\operatorname{ran}\left(B_{2} \gamma\left(\lambda_{0}\right)^{*}\right) \subset \operatorname{ran}\left(1-B_{2} M\left(\lambda_{0}\right) B_{1}\right) .
$$

Let $\psi \in \operatorname{ran}\left(B_{2} \gamma\left(\lambda_{0}\right)^{*}\right)$. Then (2.4), (iv)-(v) and $B \subset B_{1} B_{2}$ yield

$$
\psi \in \operatorname{ran}\left(B_{2}\left\lceil\operatorname{ran} \Gamma_{1}\right) \subset \operatorname{ran} \Gamma_{0} \cap \operatorname{dom} B_{1} .\right.
$$


Consider $\varphi:=\left(1-B_{2} \overline{M\left(\lambda_{0}\right) B_{1}}\right)^{-1} \psi$, which is well-defined by (i) and observe that

$$
\varphi-\psi=B_{2} \overline{M\left(\lambda_{0}\right) B_{1}} \varphi \in \operatorname{ran} \Gamma_{0} \cap \operatorname{dom} B_{1}
$$

by (ii). Combining (2.8)-(2.9) we conclude $\varphi \in \operatorname{ran} \Gamma_{0} \cap \operatorname{dom} B_{1}$ and now (iii) shows $B_{1} \varphi \in \operatorname{ran} \Gamma_{0}=\operatorname{dom} M\left(\lambda_{0}\right)$. Therefore (2.9) can be written as

$$
\left(1-B_{2} M\left(\lambda_{0}\right) B_{1}\right) \varphi=\psi
$$

and hence (2.7) holds.

Step 2. We will now prove that the operator $A_{[B]}$ in (2.5) is self-adjoint in $\mathcal{H}$. Note first that for $f, g \in \operatorname{dom} A_{[B]}$ one has

$$
\begin{aligned}
\left(A_{[B]} f, g\right)_{\mathcal{H}}-\left(f, A_{[B]} g\right)_{\mathcal{H}} & =(T f, g)_{\mathcal{H}}-(f, T g)_{\mathcal{H}} \\
& =\left(\Gamma_{1} f, \Gamma_{0} g\right)_{\mathcal{G}}-\left(\Gamma_{0} f, \Gamma_{1} g\right)_{\mathcal{G}} \\
& =\left(\Gamma_{1} f, B \Gamma_{1} g\right)_{\mathcal{G}}-\left(B \Gamma_{1} f, \Gamma_{1} g\right)_{\mathcal{G}} \\
& =0
\end{aligned}
$$

by the abstract second Green identity (2.1) and the symmetry of $B$ in $\mathcal{G}$. Therefore $A_{[B]}$ is symmetric in $\mathcal{H}$ and hence it suffices to show that

$$
\operatorname{ran}\left(A_{[B]}-\lambda_{0}\right)=\mathcal{H} \text {. }
$$

Fix $h \in \mathcal{H}$. By (2.4), (v) and $B \subset B_{1} B_{2}$, the element $B_{2} \gamma\left(\lambda_{0}\right)^{*} h$ is well-defined and according to (2.7) there exists some $g \in \operatorname{dom}\left(B_{2} M\left(\lambda_{0}\right) B_{1}\right)$ such that

$$
B_{2} \gamma\left(\lambda_{0}\right)^{*} h=\left(1-B_{2} M\left(\lambda_{0}\right) B_{1}\right) g .
$$

Consider

$$
f:=\left(A_{0}-\lambda_{0}\right)^{-1} h+\gamma\left(\lambda_{0}\right) B_{1} g
$$

and note that $\left(T-\lambda_{0}\right) f=h$ since $\operatorname{ran} \gamma\left(\lambda_{0}\right)=\operatorname{ker}\left(T-\lambda_{0}\right)$; cf. (2.3). We claim that $f \in \operatorname{dom} A_{[B]}$. In fact, since $\operatorname{dom} A_{0}=\operatorname{ker} \Gamma_{0}$ it follows from (2.4), the definition of the $\gamma$-field and the Weyl function that

$$
\Gamma_{0} f=B_{1} g \quad \text { and } \quad \Gamma_{1} f=\gamma\left(\lambda_{0}\right)^{*} h+M\left(\lambda_{0}\right) B_{1} g .
$$

Making use of condition (v) and $B \subset B_{1} B_{2}$ we then conclude

$$
B \Gamma_{1} f=B_{1}\left(B_{2} \gamma\left(\lambda_{0}\right)^{*} h+B_{2} M\left(\lambda_{0}\right) B_{1} g\right)=B_{1} g=\Gamma_{0} f
$$

from (2.11) and (2.12). Hence $f \in \operatorname{dom} A_{[B]}$ and $\left(A_{[B]}-\lambda_{0}\right) f=\left(T-\lambda_{0}\right) f=h$. Thus, (2.10) holds and therefore $A_{[B]}$ is self-adjoint in $\mathcal{H}$.

Step 3. In this step we show that

$$
\operatorname{ker}\left(1-B_{2} M(\lambda) B_{1}\right)=\{0\}, \quad \lambda \in \rho\left(A_{0}\right) \cap \rho\left(A_{[B]}\right) .
$$

In fact, for $\varphi \in \operatorname{ker}\left(1-B_{2} M(\lambda) B_{1}\right)$ one has $\varphi=B_{2} M(\lambda) B_{1} \varphi \in \operatorname{ran} \Gamma_{0}$ by (iv) and $\operatorname{ran} M(\lambda) \subset \operatorname{ran} \Gamma_{1}$. Making use of (iii) we find

$$
B_{1} \varphi=B_{1} B_{2} M(\lambda) B_{1} \varphi \in \operatorname{ran} \Gamma_{0} .
$$

Using the definition of the $\gamma$-field and the Weyl function and (v) we can rewrite (2.14) in the form

$$
\Gamma_{0} \gamma(\lambda) B_{1} \varphi=B \Gamma_{1} \gamma(\lambda) B_{1} \varphi
$$

which shows that $\gamma(\lambda) B_{1} \varphi \in \operatorname{dom} A_{[B]}$. Since $\operatorname{ran} \gamma(\lambda)=\operatorname{ker}(T-\lambda)$ and $\lambda \in \rho\left(A_{[B]}\right)$ we conclude

$$
\gamma(\lambda) B_{1} \varphi \in \operatorname{ker}\left(A_{[B]}-\lambda\right)=\{0\},
$$

and hence $\varphi=B_{2} \Gamma_{1} \gamma(\lambda) B_{1} \varphi=0$. We have shown (2.13). 
Step 4. For $\lambda \in \rho\left(A_{0}\right) \cap \rho\left(A_{[B]}\right)$ we prove $\operatorname{ran}\left(B_{2} \gamma(\bar{\lambda})^{*}\right) \subset \operatorname{ran}\left(1-B_{2} M(\lambda) B_{1}\right)$ and the resolvent formula (2.6). For $h \in \mathcal{H}$ define

$$
f_{B}:=\left(A_{[B]}-\lambda\right)^{-1} h \quad \text { and } \quad f_{0}:=\left(A_{0}-\lambda\right)^{-1} h .
$$

Then we have $f_{B}-f_{0} \in \operatorname{ker}(T-\lambda)$ and hence

$$
\gamma(\lambda) \Gamma_{0}\left(f_{B}-f_{0}\right)=f_{B}-f_{0} .
$$

Furthermore, the definitions of $A_{0}, A_{[B]}$ and (2.4) show

$$
\Gamma_{0} f_{0}=0, \quad \Gamma_{1} f_{0}=\gamma(\bar{\lambda})^{*} h, \quad \text { and } \quad \Gamma_{0} f_{B}=B \Gamma_{1} f_{B} .
$$

The element $B_{2} M(\lambda) B_{1} B_{2} \Gamma_{1} f_{B}$ is well-defined by (iii)-(v) and using (2.17) we obtain

$$
\begin{aligned}
\left(1-B_{2} M(\lambda) B_{1}\right) B_{2} \Gamma_{1} f_{B} & =B_{2} \Gamma_{1} f_{B}-B_{2} M(\lambda) \Gamma_{0} f_{B} \\
& =B_{2} \Gamma_{1} f_{B}-B_{2} M(\lambda) \Gamma_{0}\left(f_{B}-f_{0}\right) \\
& =B_{2} \Gamma_{1} f_{B}-B_{2} \Gamma_{1}\left(f_{B}-f_{0}\right) \\
& =B_{2} \gamma(\bar{\lambda})^{*} h .
\end{aligned}
$$

Since $1-B_{2} M(\lambda) B_{1}$ is invertible according to (2.13) we conclude

$$
B_{2} \Gamma_{1} f_{B}=\left(1-B_{2} M(\lambda) B_{1}\right)^{-1} B_{2} \gamma(\bar{\lambda})^{*} h .
$$

Using again $\Gamma_{0}\left(f_{B}-f_{0}\right)=B \Gamma_{1} f_{B}=B_{1} B_{2} \Gamma_{1} f_{B}$ from (2.17) as well as (2.16) leads to

$$
f_{B}-f_{0}=\gamma(\lambda) B_{1}\left(1-B_{2} M(\lambda) B_{1}\right)^{-1} B_{2} \gamma(\bar{\lambda})^{*} h .
$$

Now the Krein type resolvent formula (2.6) follows from (2.15).

\section{An eXAmple: Laplacians on the HalF-SPACE With Singular Robin BOUNDARY CONDITIONS}

In this section we illustrate our abstract techniques from the previous section by applying Corollary 2.3 to an explicit boundary value problem. On the upper halfspace $\mathbb{R}_{+}^{d}=\left\{x \in \mathbb{R}^{d}: x_{d}>0\right\}$ in $d \geq 2$ dimensions we consider the Laplacian with Robin boundary conditions $\tau_{N} f=\alpha \tau_{D} f$ on $\partial \mathbb{R}_{+}^{d} \simeq \mathbb{R}^{d-1}$ involving an unbounded parameter function $\alpha: \mathbb{R}^{d-1} \rightarrow \mathbb{R}$. Here $\tau_{D}$ and $\tau_{N}$ denote the Dirichlet and Neumann trace operator, respectively.

In order to construct a suitable quasi boundary triple consider the operators

$$
T f=-\Delta f, \quad \operatorname{dom} T=\left\{f \in H^{3 / 2}\left(\mathbb{R}_{+}^{d}\right): \Delta f \in L^{2}\left(\mathbb{R}_{+}^{d}\right)\right\},
$$

and

$$
S f=-\Delta f, \quad \operatorname{dom} S=\left\{f \in H^{2}\left(\mathbb{R}_{+}^{d}\right): \tau_{D} f=\tau_{N} f=0\right\},
$$

as well as the boundary mappings

$$
\Gamma_{0} f=\tau_{N} f \quad \text { and } \quad \Gamma_{1} f=\tau_{D} f, \quad f \in \operatorname{dom} T .
$$

The following proposition is essentially a consequence of the properties of the Dirichlet and Neumann trace operators and can be proved with standard techniques; cf. [3. Proposition 4.6]. The form of the Weyl function follows from [20, (9.65)]. 
Proposition 3.1. Let $S, T, \Gamma_{0}$ and $\Gamma_{1}$ be as above. Then $\left\{L^{2}\left(\mathbb{R}^{d-1}\right), \Gamma_{0}, \Gamma_{1}\right\}$ is a quasi boundary triple for $T \subset S^{*}$ such that

$$
\operatorname{ran} \Gamma_{0}=L^{2}\left(\mathbb{R}^{d-1}\right) \quad \text { and } \quad \operatorname{ran} \Gamma_{1}=H^{1}\left(\mathbb{R}^{d-1}\right) .
$$

Furthermore, $A_{0}=T \uparrow \operatorname{ker} \Gamma_{0}$ coincides with the Neumann Laplacian

$$
A_{N} f=-\Delta f, \quad \operatorname{dom} A_{N}=\left\{f \in H^{2}\left(\mathbb{R}_{+}^{d}\right): \tau_{N} f=0\right\},
$$

and the corresponding Weyl function is given by

$$
M(\lambda)=\left(-\Delta_{\mathbb{R}^{d-1}}-\lambda\right)^{-\frac{1}{2}}, \quad \lambda \in \mathbb{C} \backslash[0, \infty),
$$

where $\Delta_{\mathbb{R}^{d-1}}$ denotes the self-adjoint Laplacian in $L^{2}\left(\mathbb{R}^{d-1}\right)$ with domain $H^{2}\left(\mathbb{R}^{d-1}\right)$.

It follows from dom $\Delta_{\mathbb{R}^{d-1}}^{\frac{1}{2}}=H^{1}\left(\mathbb{R}^{d-1}\right)$, the continuity of the natural embedding $H^{1}\left(\mathbb{R}^{d-1}\right) \hookrightarrow H^{s}\left(\mathbb{R}^{d-1}\right)$ for $s \leq 1$ and (3.1) that

$$
M_{2, s}(\lambda): L^{2}\left(\mathbb{R}^{d-1}\right) \rightarrow H^{s}\left(\mathbb{R}^{d-1}\right), \quad \varphi \mapsto M_{2, s}(\lambda) \varphi:=M(\lambda) \varphi,
$$

is a bounded operator for every $s \leq 1$. Moreover, the next lemma shows that the values $M(\lambda)$ of the Weyl function also induce densely defined and bounded operators from $L^{p}\left(\mathbb{R}^{d-1}\right)$ into $H^{s}\left(\mathbb{R}^{d-1}\right)$ for certain values of $p$ and $s$. This is essentially a consequence of the mapping properties of the resolvent of the Laplacian on $\mathbb{R}^{d-1}$; for the convenience of the reader we provide a short proof.

Lemma 3.2. Let $M$ be the Weyl function of the quasi boundary triple in Proposition 3.1. For $\lambda \in \mathbb{C} \backslash[0, \infty), p \in[1,2)$ and $s<1-(d-1)\left(\frac{1}{p}-\frac{1}{2}\right)$ the restriction

$$
M_{p, s}(\lambda): L^{p}\left(\mathbb{R}^{d-1}\right) \rightarrow H^{s}\left(\mathbb{R}^{d-1}\right), \quad \varphi \mapsto M_{p, s}(\lambda) \varphi:=M(\lambda) \varphi,
$$

with $\operatorname{dom} M_{p, s}(\lambda)=L^{p}\left(\mathbb{R}^{d-1}\right) \cap L^{2}\left(\mathbb{R}^{d-1}\right)$ is a densely defined and bounded operator.

Proof. Denote by $\mathcal{F}$ the Fourier transform in $L^{2}\left(\mathbb{R}^{d-1}\right)$. Then it follows from (3.1) that for every $\varphi \in L^{2}\left(\mathbb{R}^{d-1}\right)$ we get

$$
(\mathcal{F} M(\lambda) \varphi)(\xi)=\left(|\xi|^{2}-\lambda\right)^{-\frac{1}{2}}(\mathcal{F} \varphi)(\xi), \quad \xi \in \mathbb{R}^{d-1} .
$$

Fix $r>0$ and choose a constant $C_{r}>0$ such that

$$
\frac{\left(1+|\xi|^{2}\right)^{s}}{\left.|| \xi\right|^{2}-\lambda \mid} \leq C_{r} \begin{cases}1, & \xi \in B_{r}, \\ |\xi|^{-(2-2 s)}, & \xi \in \mathbb{R}^{d-1} \backslash B_{r},\end{cases}
$$

where $B_{r}$ is the open ball with radius $r$ centered at 0 . Then for every function $\varphi \in L^{p}\left(\mathbb{R}^{d-1}\right) \cap L^{2}\left(\mathbb{R}^{d-1}\right)$ one has the estimate

$$
\begin{aligned}
\|M(\lambda) \varphi\|_{H^{s}\left(\mathbb{R}^{d-1}\right)}^{2} & =\int_{\mathbb{R}^{d-1}}\left(1+|\xi|^{2}\right)^{s}|(\mathcal{F} M(\lambda) \varphi)(\xi)|^{2} d \xi \\
& =\int_{\mathbb{R}^{d-1}} \frac{\left(1+|\xi|^{2}\right)^{s}}{\left.|| \xi\right|^{2}-\lambda \mid}|(\mathcal{F} \varphi)(\xi)|^{2} d \xi \\
& \leq C_{r}\left(\int_{B_{r}}|(\mathcal{F} \varphi)(\xi)|^{2} d \xi+\int_{\mathbb{R}^{d-1} \backslash B_{r}} \frac{|(\mathcal{F} \varphi)(\xi)|^{2}}{|\xi|^{2-2 s}} d \xi\right) .
\end{aligned}
$$

Using the Hölder inequality with the coefficients $\frac{p}{2-p}$ and $\frac{p}{2(p-1)}$ we obtain for the first integral

$$
\int_{B_{r}}|(\mathcal{F} \varphi)(\xi)|^{2} d \xi \leq\left|B_{r}\right|^{\frac{2-p}{p}}\|\mathcal{F} \varphi\|_{L^{\frac{p}{p-1}}}^{2}\left(\mathbb{R}^{d-1}\right)
$$


and for the second integral

$$
\int_{\mathbb{R}^{d-1} \backslash B_{r}} \frac{|(\mathcal{F} \varphi)(\xi)|^{2}}{|\xi|^{2-2 s}} d \xi \leq\left(\int_{\mathbb{R}^{d-1} \backslash B_{r}}|\xi|^{-\frac{(2-2 s) p}{2-p}} d \xi\right)^{\frac{2-p}{p}}\|\mathcal{F} \varphi\|_{L^{\frac{p}{p-1}}\left(\mathbb{R}^{d-1}\right)}^{2}
$$

As $s<1-(d-1)\left(\frac{1}{p}-\frac{1}{2}\right)$ by assumption, we have $\frac{(2-2 s) p}{2-p}>d-1$ and hence the integral on the right hand side of (3.5) is finite. Furthermore, since the Fourier transform $\mathcal{F}$ is bounded from $L^{p}\left(\mathbb{R}^{d-1}\right)$ into $L^{\frac{p}{p-1}}\left(\mathbb{R}^{d-1}\right)$ it follows from (3.4) and (3.5) that (3.3) can finally be estimated by

$$
\|M(\lambda) \varphi\|_{H^{s}\left(\mathbb{R}^{d-1}\right)}^{2} \leq C^{\prime}\|\varphi\|_{L^{p}\left(\mathbb{R}^{d-1}\right)}^{2}, \quad \varphi \in L^{p}\left(\mathbb{R}^{d-1}\right) \cap L^{2}\left(\mathbb{R}^{d-1}\right),
$$

with some constant $C^{\prime}>0$. This completes the proof of Lemma 3.2 .

The following lemma provides two important technical properties of the parameter function $\alpha$, which will be useful in the proof of Theorem 3.5

Lemma 3.3. Let $\alpha \in L^{p}\left(\mathbb{R}^{d-1}\right)+L^{\infty}\left(\mathbb{R}^{d-1}\right)$ for some $p>2$. Then for every $t \in(0,1]$ one has

$$
|\alpha|^{t} \in L^{\frac{p}{t}}\left(\mathbb{R}^{d-1}\right)+L^{\infty}\left(\mathbb{R}^{d-1}\right)
$$

and there exists a constant $C_{\alpha}>0$ such that

$$
\left\||\alpha|^{t} \varphi\right\|_{L^{2}\left(\mathbb{R}^{d-1}\right)} \leq C_{\alpha}\|\varphi\|_{H^{\frac{t(d-1)}{p}}}\left(\mathbb{R}^{d-1}\right)
$$

holds for every $\varphi \in H^{\frac{t(d-1)}{p}}\left(\mathbb{R}^{d-1}\right)$.

Proof. Decompose $\alpha=\alpha_{p}+\alpha_{\infty}$ for $\alpha_{p} \in L^{p}\left(\mathbb{R}^{d-1}\right)$ and $\alpha_{\infty} \in L^{\infty}\left(\mathbb{R}^{d-1}\right)$ and define the functions

$$
\beta_{\frac{p}{t}}(x)=\left\{\begin{array}{ll}
|\alpha(x)|^{t}, & x \in K, \\
0, & x \notin K,
\end{array} \quad \text { and } \quad \beta_{\infty}(x)= \begin{cases}0, & x \in K, \\
|\alpha(x)|^{t}, & x \notin K,\end{cases}\right.
$$

where $K=\left\{x \in \mathbb{R}^{d-1}:|\alpha(x)|>\left\|\alpha_{\infty}\right\|_{L^{\infty}\left(\mathbb{R}^{d-1}\right)}+1\right\}$. Note that $K$ is contained in the set $\left\{x \in \mathbb{R}^{d-1}:\left|\alpha_{p}(x)\right|>1\right\}$, which has finite measure since $\alpha_{p} \in L^{p}\left(\mathbb{R}^{d-1}\right)$. Hence $K$ has finite measure as well. It is obvious that $\beta_{\infty} \in L^{\infty}\left(\mathbb{R}^{d-1}\right)$ and moreover, the estimate

$$
\begin{aligned}
\int_{\mathbb{R}^{d-1}}\left|\beta_{\frac{p}{t}}(x)\right|^{\frac{p}{t}} d x & =\int_{K}\left|\alpha_{p}(x)+\alpha_{\infty}(x)\right|^{p} d x \\
& \leq 2^{p-1}\left(\int_{K}\left|\alpha_{p}(x)\right|^{p} d x+\int_{K}\left|\alpha_{\infty}(x)\right|^{p} d x\right) \\
& \leq 2^{p-1}\left(\left\|\alpha_{p}\right\|_{L^{p}\left(\mathbb{R}^{d-1}\right)}^{p}+|K|\left\|\alpha_{\infty}\right\|_{L^{\infty}\left(\mathbb{R}^{d-1}\right)}^{p}\right)
\end{aligned}
$$

shows that $\beta_{\frac{p}{t}} \in L^{\frac{p}{t}}\left(\mathbb{R}^{d-1}\right)$. Hence $|\alpha|^{t}=\beta_{\frac{p}{t}}+\beta_{\infty} \in L^{\frac{p}{t}}\left(\mathbb{R}^{d-1}\right)+L^{\infty}\left(\mathbb{R}^{d-1}\right)$.

Using the decomposition $|\alpha|^{t}=\beta_{\frac{p}{t}}+\beta_{\infty}$ from above, we can prove (3.7) by estimating both terms separately. For the bounded part $\beta_{\infty}$ it is clear that

$$
\begin{aligned}
\left\|\beta_{\infty} \varphi\right\|_{L^{2}\left(\mathbb{R}^{d-1}\right)} & \leq\left\|\beta_{\infty}\right\|_{L^{\infty}\left(\mathbb{R}^{d-1}\right)}\|\varphi\|_{L^{2}\left(\mathbb{R}^{d-1}\right)} \\
& \leq\left\|\beta_{\infty}\right\|_{L^{\infty}\left(\mathbb{R}^{d-1}\right)}\|\varphi\|_{H^{\frac{t(d-1)}{p}}\left(\mathbb{R}^{d-1}\right)}
\end{aligned}
$$


holds for all $\varphi \in H^{\frac{t(d-1)}{p}}\left(\mathbb{R}^{d-1}\right)$. For the estimate of the unbounded part $\beta \frac{p}{t}$ note first that by assumption we ensured $p>2 \geq 2 t$. Hence the Hölder inequality with the coefficients $\frac{p}{2 t}$ and $\frac{p}{p-2 t}$ yields

$$
\begin{aligned}
\left\|\beta_{\frac{p}{t}} \varphi\right\|_{L^{2}\left(\mathbb{R}^{d-1}\right)} & \leq\left\|\beta_{\frac{p}{t}}\right\|_{L^{\frac{p}{t}}\left(\mathbb{R}^{d-1}\right)}\|\varphi\|_{L^{\frac{2 p}{p-2 t}}\left(\mathbb{R}^{d-1}\right)} \\
& \leq C\left\|\beta_{\frac{p}{t}}\right\|_{L^{\frac{p}{t}}\left(\mathbb{R}^{d-1}\right)}\|\varphi\|_{H^{\frac{t(d-1)}{p}}\left(\mathbb{R}^{d-1}\right)}
\end{aligned}
$$

for all $\varphi \in H^{\frac{t(d-1)}{p}}\left(\mathbb{R}^{d-1}\right)$, where $C>0$ is the constant of the Sobolev embedding theorem [8, Theorem 8.12.4 Case I]. Combining (3.8) and (3.9) leads to the estimate (3.7).

In the next lemma we recall a simple estimate for functions $f \in H^{1}\left(\mathbb{R}_{+}^{d}\right)$. For the convenience of the reader we provide a short proof.

Lemma 3.4. Let $s \in[0,1)$. Then for every $\varepsilon>0$ there exists a constant $C_{\varepsilon}>0$ such that

$$
\|f\|_{H^{s}\left(\mathbb{R}_{+}^{d}\right)}^{2} \leq \varepsilon\|\nabla f\|_{L^{2}\left(\mathbb{R}_{+}^{d}, \mathbb{C}^{d}\right)}^{2}+C_{\varepsilon}\|f\|_{L^{2}\left(\mathbb{R}_{+}^{d}\right)}^{2}
$$

holds for every $f \in H^{1}\left(\mathbb{R}_{+}^{d}\right)$.

Proof. Recall from [35, §3 Theorem 5] that there exists an extension operator $E$ : $L^{2}\left(\mathbb{R}_{+}^{d}\right) \rightarrow L^{2}\left(\mathbb{R}^{d}\right)$ which satisfies

$$
\|E g\|_{L^{2}\left(\mathbb{R}^{d}\right)} \leq c_{E}\|g\|_{L^{2}\left(\mathbb{R}_{+}^{d}\right)} \quad \text { and } \quad\|E f\|_{H^{1}\left(\mathbb{R}^{d}\right)} \leq c_{E}\|f\|_{H^{1}\left(\mathbb{R}_{+}^{d}\right)}
$$

for some $c_{E}>0$ and all $g \in L^{2}\left(\mathbb{R}_{+}^{d}\right), f \in H^{1}\left(\mathbb{R}_{+}^{d}\right)$. From [22, Theorem 3.30] we can conclude that for $\varepsilon^{\prime}>0$ there exists $C_{\varepsilon^{\prime}}>0$ such that

$$
\|f\|_{H^{s}\left(\mathbb{R}_{+}^{d}\right)} \leq\|E f\|_{H^{s}\left(\mathbb{R}^{d}\right)} \leq \varepsilon^{\prime}\|E f\|_{H^{1}\left(\mathbb{R}^{d}\right)}+C_{\varepsilon^{\prime}}\|E f\|_{L^{2}\left(\mathbb{R}^{d}\right)}
$$

for every $f \in H^{1}\left(\mathbb{R}_{+}^{d}\right)$. Together with (3.11) this leads to (3.10).

After these preparations we are now ready to formulate and prove the main theorem of this section.

Theorem 3.5. Let $\alpha \in L^{p}\left(\mathbb{R}^{d-1}\right)+L^{\infty}\left(\mathbb{R}^{d-1}\right)$ be a real-valued function and assume that $p>\frac{4}{3}(d-1)$ if $d \geq 3$ and $p>2$ if $d=2$. Then the Robin-Laplacian

$$
A_{\alpha} f=-\Delta f, \quad \operatorname{dom} A_{\alpha}=\left\{f \in H^{3 / 2}\left(\mathbb{R}_{+}^{d}\right): \begin{array}{c}
\Delta f \in L^{2}\left(\mathbb{R}_{+}^{d}\right), \\
\alpha \tau_{D} f=\tau_{N} f
\end{array}\right\},
$$

is self-adjoint in $L^{2}\left(\mathbb{R}_{+}^{d}\right)$ and for every $\lambda \in \rho\left(A_{\alpha}\right) \backslash[0, \infty)$ the Krein type resolvent formula

$$
\begin{aligned}
& \left(A_{\alpha}-\lambda\right)^{-1}-\left(A_{N}-\lambda\right)^{-1} \\
& \quad=\gamma(\lambda) \operatorname{sgn}(\alpha)|\alpha|^{\frac{1}{3}}\left(1+|\alpha|^{\frac{2}{3}}\left(-\Delta_{\mathbb{R}^{d-1}}-\lambda\right)^{-\frac{1}{2}} \operatorname{sgn}(\alpha)|\alpha|^{\frac{1}{3}}\right)^{-1}|\alpha|^{\frac{2}{3}} \gamma(\bar{\lambda})^{*}
\end{aligned}
$$

is valid.

Proof. This theorem is a consequence of Corollary 2.3 and hence in the following it will be shown that its assumptions are satisfied. We start by defining the multiplication operator

$$
B \varphi=\alpha \varphi, \quad \operatorname{dom} B=H^{1}\left(\mathbb{R}^{d-1}\right),
$$


in the boundary space $L^{2}\left(\mathbb{R}^{d-1}\right)$. Note that by assumption we have $p>2$ as well as $p>t(d-1)$, for every $t \in(0,1]$ in any dimension $d \geq 2$. Hence by Lemma 3.3 the estimate

$$
\left\||\alpha|^{t} \varphi\right\|_{L^{2}\left(\mathbb{R}^{d-1}\right)} \leq C_{\alpha}\|\varphi\|_{H^{\frac{t(d-1)}{p}}\left(\mathbb{R}^{d-1}\right)} \leq C_{\alpha}\|\varphi\|_{H^{1}\left(\mathbb{R}^{d-1}\right)}
$$

holds for every $\varphi \in H^{1}\left(\mathbb{R}^{d-1}\right)$ and the operator $B$ is well-defined. Clearly the first inequality in (3.13) also holds for $\varphi \in H^{\frac{t(d-1)}{p}}\left(\mathbb{R}^{d-1}\right)$. Next we decompose $B$ into

$$
\begin{array}{ll}
B_{1} \varphi=\operatorname{sgn}(\alpha)|\alpha|^{\frac{1}{3}} \varphi, & \operatorname{dom} B_{1}=\left\{\varphi \in L^{2}\left(\mathbb{R}^{d-1}\right):|\alpha|^{\frac{1}{3}} \varphi \in L^{2}\left(\mathbb{R}^{d-1}\right)\right\}, \\
B_{2} \varphi=|\alpha|^{\frac{2}{3}} \varphi, & \operatorname{dom} B_{2}=\left\{\varphi \in L^{2}\left(\mathbb{R}^{d-1}\right):|\alpha|^{\frac{2}{3}} \varphi \in L^{2}\left(\mathbb{R}^{d-1}\right)\right\} .
\end{array}
$$

Using the first estimate in (3.13) it follows that every $\varphi \in \operatorname{dom} B=H^{1}\left(\mathbb{R}^{d-1}\right)$ satisfies $\varphi \in \operatorname{dom} B_{2}$ and $B_{2} \varphi \in \operatorname{dom} B_{1}$, and hence the operator inclusion $B \subset$ $B_{1} B_{2}$ holds.

For the operators $B, B_{1}$ and $B_{2}$ we now verify the assumptions in Corollary 2.3 First of all, since $\alpha$ is real-valued, it is clear that the operator $B$ is symmetric in $L^{2}\left(\mathbb{R}^{d-1}\right)$. Moreover, $\operatorname{ran} \Gamma_{0}=L^{2}\left(\mathbb{R}^{d-1}\right)$ as well as $\operatorname{ran} \Gamma_{1}=H^{1}\left(\mathbb{R}^{d-1}\right)$ holds by Proposition 3.1 and hence also assumption (iii) in Corollary 2.3 is fulfilled. Therefore, it remains to choose a suitable $\lambda_{0} \in \rho\left(A_{N}\right) \cap \mathbb{R}=(-\infty, 0)$ such that the assumptions (i) and (ii) are satisfied.

Using again (3.13), the boundedness of the Dirichlet trace operator

$$
\tau_{D}: H^{\frac{2(d-1)}{3 p}+\frac{1}{2}}\left(\mathbb{R}_{+}^{d}\right) \rightarrow H^{\frac{2(d-1)}{3 p}}\left(\mathbb{R}^{d-1}\right),
$$

and Lemma 3.4. we find a constant $c_{1}>0$ such that

$$
\begin{aligned}
\left\||\alpha|^{\frac{2}{3}} \tau_{D} g\right\|_{L^{2}\left(\mathbb{R}^{d-1}\right)}^{2} & \leq C_{\alpha}^{2}\left\|\tau_{D} g\right\|_{H}^{2}{ }_{H}^{\frac{2(d-1)}{3 p}}\left(\mathbb{R}^{d-1}\right) \\
& \leq C_{\alpha}^{2}\left\|\tau_{D}\right\|^{2}\|g\|_{H^{\frac{2(d-1)}{3 p}}+\frac{1}{2}}^{2}\left(\mathbb{R}_{+}^{d}\right) \\
& \leq \frac{1}{2}\|\nabla g\|_{L^{2}\left(\mathbb{R}_{+}^{d}, \mathbb{C}^{d}\right)}^{2}+c_{1}\|g\|_{L^{2}\left(\mathbb{R}_{+}^{d}\right)}^{2}
\end{aligned}
$$

holds for all $g \in H^{1}\left(\mathbb{R}_{+}^{d}\right)$. In the last step it was crucial that $\frac{2(d-1)}{3 p}+\frac{1}{2}<1$, which is equivalent to $p>\frac{4}{3}(d-1)$ and is fulfilled by the assumptions on $p$ in every dimension $d \geq 2$. In the same way we find a constant $c_{2}>0$ such that

$$
\left\||\alpha|^{\frac{1}{3}} \tau_{D} g\right\|_{L^{2}\left(\mathbb{R}^{d-1}\right)}^{2} \leq \frac{1}{2}\|\nabla g\|_{L^{2}\left(\mathbb{R}_{+}^{d}, \mathbb{C}^{d}\right)}^{2}+c_{2}\|g\|_{L^{2}\left(\mathbb{R}_{+}^{d}\right)}^{2}
$$

holds for all $g \in H^{1}\left(\mathbb{R}_{+}^{d}\right)$. For the choice $\lambda_{0}:=-2 \max \left\{c_{1}, c_{2}\right\} \in \rho\left(A_{N}\right)$, the estimates (3.14) and (3.15) turn into

$$
\begin{aligned}
\left\||\alpha|^{\frac{2}{3}} \tau_{D} g\right\|_{L^{2}\left(\mathbb{R}^{d-1}\right)}^{2} & \leq \frac{1}{2}\left(\|\nabla g\|_{L^{2}\left(\mathbb{R}_{+}^{d}, \mathbb{C}^{d}\right)}^{2}-\lambda_{0}\|g\|_{L^{2}\left(\mathbb{R}_{+}^{d}\right)}^{2}\right), \\
\left\||\alpha|^{\frac{1}{3}} \tau_{D} g\right\|_{L^{2}\left(\mathbb{R}^{d-1}\right)}^{2} & \leq \frac{1}{2}\left(\|\nabla g\|_{L^{2}\left(\mathbb{R}_{+}^{d}, \mathbb{C}^{d}\right)}^{2}-\lambda_{0}\|g\|_{L^{2}\left(\mathbb{R}_{+}^{d}\right)}^{2}\right),
\end{aligned}
$$

for all $g \in H^{1}\left(\mathbb{R}_{+}^{d}\right)$.

Assumption (ii). In order to check $\operatorname{ran}\left(B_{2} \overline{M\left(\lambda_{0}\right) B_{1}}\right) \subset \operatorname{dom} B_{1}$ we have to show $|\alpha|^{\frac{1}{3}} B_{2} \overline{M\left(\lambda_{0}\right) B_{1}} \varphi=|\alpha| \overline{M\left(\lambda_{0}\right) B_{1}} \varphi \in L^{2}\left(\mathbb{R}^{d-1}\right)$ for all functions $\varphi \in \operatorname{dom}\left(B_{2} \overline{M\left(\lambda_{0}\right) B_{1}}\right)$.

Using (3.13) it suffices to verify $\overline{M\left(\lambda_{0}\right) B_{1}} \varphi \in H^{\frac{d-1}{p}}\left(\mathbb{R}^{d-1}\right)$. 
First consider $\varphi \in \operatorname{dom}\left(M\left(\lambda_{0}\right) B_{1}\right)$ and choose $\beta_{3 p} \in L^{3 p}\left(\mathbb{R}^{d-1}\right)$ and $\beta_{\infty} \in$ $L^{\infty}\left(\mathbb{R}^{d-1}\right)$ such that $\operatorname{sgn}(\alpha)|\alpha|^{\frac{1}{3}}=\beta_{3 p}+\beta_{\infty}$; cf. (3.6). Then by the boundedness of the Weyl function in Lemma 3.2 and (3.2) we obtain

$$
\begin{aligned}
\| M\left(\lambda_{0}\right) & B_{1} \varphi \|_{H^{\frac{d-1}{p}}\left(\mathbb{R}^{d-1}\right)} \\
& \leq\left\|M\left(\lambda_{0}\right) \beta_{3 p} \varphi\right\|_{H^{\frac{d-1}{p}}\left(\mathbb{R}^{d-1}\right)}+\left\|M\left(\lambda_{0}\right) \beta_{\infty} \varphi\right\|_{H^{\frac{d-1}{p}}\left(\mathbb{R}^{d-1}\right)} \\
& \leq c\left(\left\|\beta_{3 p} \varphi\right\|_{L^{\frac{6 p}{3 p+2}}\left(\mathbb{R}^{d-1}\right)}+\left\|\beta_{\infty} \varphi\right\|_{L^{2}\left(\mathbb{R}^{d-1}\right)}\right) \\
& \leq c\left(\left\|\beta_{3 p}\right\|_{L^{3 p}\left(\mathbb{R}^{d-1}\right)}+\left\|\beta_{\infty}\right\|_{L^{\infty}\left(\mathbb{R}^{d-1}\right)}\right)\|\varphi\|_{L^{2}\left(\mathbb{R}^{d-1}\right)},
\end{aligned}
$$

where Lemma 3.2 was used in the penultimate inequality with $s$ and $p$ replaced by $\frac{d-1}{p}$ and $\frac{6 p}{3 p+2}$, respectively, which is possible since $p>\frac{4}{3}(d-1)$ holds by assumption for every dimension $d \geq 2$. Furthermore, in the last estimate the Hölder inequality with the exponents $\frac{3 p+2}{2}$ and $\frac{3 p+2}{3 p}$ was used.

Now let $\varphi \in \operatorname{dom}\left(\overline{M\left(\lambda_{0}\right) B_{1}}\right)$ and pick a sequence $\left(\varphi_{n}\right) \subset \operatorname{dom}\left(M\left(\lambda_{0}\right) B_{1}\right)$ such that $\varphi_{n} \rightarrow \varphi$ and $M\left(\lambda_{0}\right) B_{1} \varphi_{n} \rightarrow \overline{M\left(\lambda_{0}\right) B_{1}} \varphi$ for $n \rightarrow \infty$ in $L^{2}\left(\mathbb{R}^{d-1}\right)$. It is clear from (3.18) that the sequence $\left(M\left(\lambda_{0}\right) B_{1} \varphi_{n}\right)$ converges in $H^{\frac{d-1}{p}}\left(\mathbb{R}^{d-1}\right)$ to an element $g \in H^{\frac{d-1}{p}}\left(\mathbb{R}^{d-1}\right)$. Hence it follows that

$$
g=\overline{M\left(\lambda_{0}\right) B_{1}} \varphi \in H^{\frac{d-1}{p}}\left(\mathbb{R}^{d-1}\right) .
$$

Therefore, assumption (ii) in Corollary 2.3 holds.

Assumption (i). We prove $1 \in \rho\left(B_{2} \overline{M\left(\lambda_{0}\right) B_{1}}\right)$ by showing that $B_{2} \overline{M\left(\lambda_{0}\right) B_{1}}$ is an everywhere defined bounded operator with norm strictly less than 1 .

For this we define the inner product

$$
(f, g)_{\lambda_{0}}:=(\nabla f, \nabla g)_{L^{2}\left(\mathbb{R}_{+}^{d}, \mathbb{C}^{d}\right)}-\lambda_{0}(f, g)_{L^{2}\left(\mathbb{R}_{+}^{d}\right)}, \quad f, g \in H^{1}\left(\mathbb{R}_{+}^{d}\right),
$$

and note that the corresponding norm is equivalent to the usual $H^{1}\left(\mathbb{R}_{+}^{d}\right)$-norm. Fix now any $\varphi \in \operatorname{dom}\left(B_{2} M\left(\lambda_{0}\right) B_{1}\right)$ and use (3.16) for $g=\gamma\left(\lambda_{0}\right) B_{1} \varphi$ to obtain the estimate

$$
\begin{aligned}
\left\|B_{2} M\left(\lambda_{0}\right) B_{1} \varphi\right\|_{L^{2}\left(\mathbb{R}^{d-1}\right)}^{2} & =\left\||\alpha|^{\frac{2}{3}} \tau_{D} \gamma\left(\lambda_{0}\right) B_{1} \varphi\right\|_{L^{2}\left(\mathbb{R}^{d-1}\right)}^{2} \\
& \leq \frac{1}{2}\left\|\gamma\left(\lambda_{0}\right) B_{1} \varphi\right\|_{\lambda_{0}}^{2} \\
& =\frac{1}{2} \sup _{h \in H^{1}\left(\mathbb{R}_{+}^{d}\right) \backslash\{0\}} \frac{\left(\gamma\left(\lambda_{0}\right) B_{1} \varphi, h\right)_{\lambda_{0}}^{2}}{\|h\|_{\lambda_{0}}^{2}} .
\end{aligned}
$$

Using the first Green identity and the properties

$$
\left(-\Delta-\lambda_{0}\right) \gamma\left(\lambda_{0}\right) B_{1} \varphi=0 \text { and } \tau_{N} \gamma\left(\lambda_{0}\right) B_{1} \varphi=B_{1} \varphi,
$$

of the $\gamma$-field, which follow immediately from its definition (2.3) and Proposition 3.1 we find

$$
\begin{aligned}
\left(\gamma\left(\lambda_{0}\right) B_{1} \varphi, h\right)_{\lambda_{0}} & =\left(\nabla \gamma\left(\lambda_{0}\right) B_{1} \varphi, \nabla h\right)_{L^{2}\left(\mathbb{R}_{+}^{d}, \mathbb{C}^{d}\right)}-\lambda_{0}\left(\gamma\left(\lambda_{0}\right) B_{1} \varphi, h\right)_{L^{2}\left(\mathbb{R}_{+}^{d}\right)} \\
& =\left(\nabla \gamma\left(\lambda_{0}\right) B_{1} \varphi, \nabla h\right)_{L^{2}\left(\mathbb{R}_{+}^{d}, \mathbb{C}^{d}\right)}+\left(\Delta \gamma\left(\lambda_{0}\right) B_{1} \varphi, h\right)_{L^{2}\left(\mathbb{R}_{+}^{d}\right)} \\
& =\left(\tau_{N} \gamma\left(\lambda_{0}\right) B_{1} \varphi, \tau_{D} h\right)_{L^{2}\left(\mathbb{R}^{d-1}\right)} \\
& =\left(B_{1} \varphi, \tau_{D} h\right)_{L^{2}\left(\mathbb{R}^{d-1}\right)}
\end{aligned}
$$


and hence

$$
\begin{aligned}
\left\|B_{2} M\left(\lambda_{0}\right) B_{1} \varphi\right\|_{L^{2}\left(\mathbb{R}^{d-1}\right)}^{2} & \leq \frac{1}{2} \sup _{h \in H^{1}\left(\mathbb{R}_{+}^{d}\right) \backslash\{0\}} \frac{\left(B_{1} \varphi, \tau_{D} h\right)_{L^{2}\left(\mathbb{R}^{d-1}\right)}^{2}}{\|h\|_{\lambda_{0}}^{2}} \\
& \leq \frac{1}{2}\|\varphi\|_{L^{2}\left(\mathbb{R}^{d-1}\right)}^{2} \sup _{h \in H^{1}\left(\mathbb{R}_{+}^{d}\right) \backslash\{0\}} \frac{\left\|\left.\alpha\right|^{\frac{1}{3}} \tau_{D} h\right\|_{L^{2}\left(\mathbb{R}^{d-1}\right)}^{2}}{\|h\|_{\lambda_{0}}^{2}} .
\end{aligned}
$$

Equation (3.17) then leads to the estimate

$$
\left\|B_{2} M\left(\lambda_{0}\right) B_{1} \varphi\right\|_{L^{2}\left(\mathbb{R}^{d-1}\right)}^{2} \leq \frac{1}{4}\|\varphi\|_{L^{2}\left(\mathbb{R}^{d-1}\right)}^{2}
$$

for any $\varphi \in \operatorname{dom}\left(B_{2} M\left(\lambda_{0}\right) B_{1}\right)$.

As $B_{2}$ is closed and (3.18) implies that $M\left(\lambda_{0}\right) B_{1}$ is bounded in $L^{2}\left(\mathbb{R}^{d-1}\right)$ it follows that $B_{2} \overline{M\left(\lambda_{0}\right) B_{1}}$ is closed in $L^{2}\left(\mathbb{R}^{d-1}\right)$ as well. Since $\overline{B_{2} M\left(\lambda_{0}\right) B_{1}}$ is everywhere defined, this however implies $\overline{B_{2} M\left(\lambda_{0}\right) B_{1}}=B_{2} \overline{M\left(\lambda_{0}\right) B_{1}}$ and hence $1 \epsilon$ $\rho\left(B_{2} \overline{M\left(\lambda_{0}\right) B_{1}}\right)$ follows from (3.19). This completes the proof of Theorem 3.5.

Remark 3.6. If one uses Corollary 2.4 instead of Corollary 2.3 in the proof of Theorem 3.5 only $\alpha \in L^{p}\left(\mathbb{R}^{d-1}\right)+L^{\infty}\left(\mathbb{R}^{d-1}\right)$ with $p>2(d-1)$ can be treated. In fact, in this situation one chooses $B_{2}=B$ to be the multiplication operator with $\alpha$ and for the estimate (3.14) (with $\alpha$ instead of $|\alpha|^{\frac{2}{3}}$ ) it is necessary to restrict to $p>2(d-1)$. Thus, for Laplacians with singular Robin boundary conditions Theorem 2.2 and Corollary 2.3 allow a larger class of boundary parameters $\alpha$ than Corollary 2.4.

Remark 3.7. A variant of Theorem 3.5 for more general elliptic second order operators on a certain class of unbounded non-smooth domains with Robin boundary conditions containing also differential or pseudodifferential operators can be found in [1. In our situation for a Robin Laplacian on $\mathbb{R}_{+}^{d}$ with an $H^{1 / 2}$-smooth real-valued

$$
\alpha \in H_{p}^{1 / 2}\left(\mathbb{R}^{d-1}\right), \quad p>2(d-1),
$$

it follows from [1, Theorem 7.2] that the operator

$$
A_{\alpha} f=-\Delta f, \quad \operatorname{dom} A_{\alpha}=\left\{f \in H^{2}\left(\mathbb{R}_{+}^{d}\right): \alpha \tau_{D} f=\tau_{N} f\right\},
$$

is self-adjoint in $L^{2}\left(\mathbb{R}_{+}^{d}\right)$.

Self-adjoint Laplacians with Robin boundary conditions can also be defined via the densely defined, symmetric form

$$
\mathfrak{a}_{\alpha}[f]=\|\nabla f\|_{L^{2}\left(\mathbb{R}^{d}, \mathbb{C}^{d}\right)}^{2}-\int_{\mathbb{R}^{d-1}} \alpha\left|\tau_{D} f\right|^{2} d x, \quad \operatorname{dom} \mathfrak{a}_{\alpha}=H^{1}\left(\mathbb{R}_{+}^{d}\right),
$$

and the first representation theorem [26, VI Theorem 2.1]. The following proposition shows that this method allows a larger class of boundary parameters $\alpha$ as Theorem 3.5. does, but leads to an operator $A_{\alpha}$ with a less regular operator domain. However, for functions $\alpha$ satisfying the stronger assumptions in Theorem 3.5, the operators in (3.21) below and in (3.12) coincide. A variant of Proposition 3.8 for bounded Lipschitz domains can be found in [18, Theorem 4.5 and Lemma 5.3].

Proposition 3.8. Let $\alpha \in L^{p}\left(\mathbb{R}^{d-1}\right)+L^{\infty}\left(\mathbb{R}^{d-1}\right)$ be a real-valued function for $p=d-1$ if $d \geq 3$ and $p>1$ if $d=2$. Then the quadratic form $\mathfrak{a}_{\alpha}$ in (3.20) 
is semibounded and closed. The corresponding self-adjoint operator in $L^{2}\left(\mathbb{R}_{+}^{d}\right)$ is given by

$$
A_{\alpha} f=-\Delta f, \quad \operatorname{dom} A_{\alpha}=\left\{f \in H^{1}\left(\mathbb{R}_{+}^{d}\right): \begin{array}{l}
\Delta f \in L^{2}\left(\mathbb{R}_{+}^{d}\right), \\
\alpha \tau_{D} f=\tau_{N} f
\end{array}\right\} .
$$

Proof. In order to prove that the form $\mathfrak{a}_{\alpha}$ is semibounded and closed we split $\mathfrak{a}_{\alpha}$ into the two quadratic forms

$$
\mathfrak{a}[f]=\|\nabla f\|_{L^{2}\left(\mathbb{R}_{+}^{d}, \mathbb{C}^{d}\right)}^{2} \quad \text { and } \quad \mathfrak{t}[f]=\int_{\mathbb{R}^{d-1}} \alpha(x)\left|\tau_{D} f(x)\right|^{2} d x
$$

with dom $\mathfrak{a}=\operatorname{dom} \mathfrak{t}=H^{1}\left(\mathbb{R}_{+}^{d}\right)$ and observe that $\mathfrak{a}$ is a densely defined, nonnegative, closed form in $L^{2}\left(\mathbb{R}^{d-1}\right)$. Now it suffices to check that $\mathfrak{t}$ is relatively bounded with respect to $\mathfrak{a}$ with relative bound $<1$, that is, for some $a \geq 0$ and $0 \leq b<1$

$$
|\mathfrak{t}[f]| \leq a\|f\|_{L^{2}\left(\mathbb{R}_{+}^{d}\right)}^{2}+b \mathfrak{a}[f], \quad f \in H^{1}\left(\mathbb{R}_{+}^{d}\right),
$$

since in this case the KLMN theorem [36, Theorem 6.24] (see also [26, VI Theorem 1.33]) yields that the form $\mathfrak{a}_{\alpha}=\mathfrak{a}-\mathfrak{t}$ is densely defined, closed, and semibounded in $L^{2}\left(\mathbb{R}^{d-1}\right)$. To verify (3.22), decompose the function $\alpha=\alpha_{p}+\alpha_{\infty}$ in the sum of $\alpha_{p} \in L^{p}\left(\mathbb{R}^{d-1}\right)$ and $\alpha_{\infty} \in L^{\infty}\left(\mathbb{R}^{d-1}\right)$, and let $\varepsilon>0$. For the unbounded part $\alpha_{p}$ choose a sufficiently large $\gamma_{\varepsilon}>0$ such that

$$
\left\|\alpha_{p}\right\|_{L^{p}\left(K_{\varepsilon}\right)} \leq \varepsilon \quad \text { with } \quad K_{\varepsilon}=\left\{x \in \mathbb{R}^{d-1}:\left|\alpha_{p}(x)\right|>\gamma_{\varepsilon}\right\},
$$

and write $\alpha_{p}$ as the sum of

$$
\alpha_{p}^{(0)}(x)=\left\{\begin{array}{ll}
0, & x \in K_{\varepsilon}, \\
\alpha_{p}(x), & x \notin K_{\varepsilon},
\end{array} \quad \text { and } \quad \alpha_{p}^{(1)}(x)= \begin{cases}\alpha_{p}(x), & x \in K_{\varepsilon}, \\
0, & x \notin K_{\varepsilon} .\end{cases}\right.
$$

With this decomposition we now estimate the form $\mathfrak{t}$ by

$$
\begin{aligned}
|\mathfrak{t}[f]| \leq \int_{\mathbb{R}^{d-1}} \mid \alpha_{\infty}(x)+ & \left.\alpha_{p}^{(0)}(x)|| \tau_{D} f(x)\right|^{2} d x \\
& +\int_{\mathbb{R}^{d-1}}\left|\alpha_{p}^{(1)}(x)\right|\left|\tau_{D} f(x)\right|^{2} d x
\end{aligned}
$$

and discuss both integrals on the right hand side of (3.24) separately. For the first integral we fix some arbitrary $s \in\left(\frac{1}{2}, 1\right)$ and use the continuity of $\tau_{D}: H^{s}\left(\mathbb{R}_{+}^{d}\right) \rightarrow$ $H^{s-\frac{1}{2}}\left(\mathbb{R}^{d-1}\right)$ as well as Lemma 3.4 to obtain

$$
\begin{aligned}
\int_{\mathbb{R}^{d-1}}\left|\alpha_{\infty}(x)+\alpha_{p}^{(0)}(x)\right|\left|\tau_{D} f(x)\right|^{2} d x \\
\leq\left(\left\|\alpha_{\infty}\right\|_{L^{\infty}\left(\mathbb{R}^{d-1}\right)}+\gamma_{\varepsilon}\right)\left\|\tau_{D} f\right\|_{L^{2}\left(\mathbb{R}^{d-1}\right)}^{2} \\
\leq c^{\prime}\|f\|_{H^{s}\left(\mathbb{R}_{+}^{d}\right)}^{2} \\
\leq c^{\prime} \varepsilon\|\nabla f\|_{L^{2}\left(\mathbb{R}_{+}^{d}, \mathbb{C}^{d}\right)}^{2}+c^{\prime} C_{\varepsilon}\|f\|_{L^{2}\left(\mathbb{R}_{+}^{d}\right)}^{2}
\end{aligned}
$$

where $c^{\prime}=\left(\left\|\alpha_{\infty}\right\|_{L^{\infty}\left(\mathbb{R}^{d-1}\right)}+\gamma_{\varepsilon}\right)\left\|\tau_{D}\right\|^{2}$ and $C_{\varepsilon}$ is the constant in Lemma 3.4. For the estimate of the second integral in (3.24) we first use the Hölder inequality and (3.23) to obtain

$$
\begin{aligned}
\int_{\mathbb{R}^{d-1}}\left|\alpha_{p}^{(1)}(x)\right|\left|\tau_{D} f(x)\right|^{2} d x & \leq\left\|\alpha_{p}^{(1)}\right\|_{L^{p}\left(\mathbb{R}^{d-1}\right)}\left\|\tau_{D} f\right\|_{L^{\frac{2 p}{p-1}}\left(\mathbb{R}^{d-1}\right)}^{2} \\
& \leq \varepsilon\left\|\tau_{D} f\right\|_{L^{\frac{2 p}{p-1}}\left(\mathbb{R}^{d-1}\right)}^{2}
\end{aligned}
$$


By the given assumptions on $p$ we can now apply the Sobolev embedding theorem [8, Theorem 8.12.4 Case I] if $d \geq 3$ and [8, Theorem 8.12.4 Case II] if $d=2$. This leads to the estimate

$$
\int_{\mathbb{R}^{d-1}}\left|\alpha_{p}^{(1)}\right|\left|\tau_{D} f(x)\right|^{2} d x \leq \varepsilon c^{\prime \prime}\left\|\tau_{D} f\right\|_{H^{\frac{1}{2}}\left(\mathbb{R}^{d-1}\right)}^{2} \leq \varepsilon c^{\prime \prime \prime}\|f\|_{H^{1}\left(\mathbb{R}_{+}^{d}\right)}^{2}
$$

with some constants $c^{\prime \prime}, c^{\prime \prime \prime}>0$. From (3.25) and (3.26) we conclude that (3.22) holds for all $b>0$ and hence it follows, in particular, that $\mathfrak{a}_{\alpha}$ closed and semibounded.

We leave it to the reader to verify that the self-adjoint operator corresponding to $\mathfrak{a}_{\alpha}$ is given by (3.21).

\section{REFERENCES}

[1] H. Abels, G. Grubb and G. Wood, Extension theory and Krein-type resolvent formulas for nonsmooth boundary value problems, J. Funct. Anal. 266 (2014) 4037-4100.

[2] W. Arendt and M. Warma, The Laplacian with Robin boundary conditions on arbitrary domains, Potential Anal. 19 (2003), 341-363.

[3] J. Behrndt and M. Langer, Boundary value problems for elliptic partial differential operators on bounded domains, J. Funct. Anal. 243 (2007), 536-565.

[4] J. Behrndt and M. Langer, Elliptic operators, Dirichlet-to-Neumann maps and quasi boundary triples, in: Operator Methods for Boundary Value Problems, London Math. Soc. Lecture Note Series 404, 2012, pp. 121-160.

[5] J. Behrndt, M. Langer, V. Lotoreichik, and J. Rohleder, Quasi boundary triples and semibounded self-adjoint extensions, Proc. Roy. Soc. Edinburgh A 147 (2017), 895-916.

[6] M. V. Berry, Hermitian boundary conditions at a Dirichlet singularity: the MarlettaRozenblum model, J. Phys. A 42 (2009), 165208, 13 pp.

[7] M. V. Berry and M. Dennis, Boundary-condition-varying circle billiards and gratings: the Dirichlet singularity, J. Phys. A 41 (2008), 135203, 23 pp.

[8] P.K. Bhattacharyya, Distributions. Generalized Functions with Applications in Sobolev Spaces, de Gruyter Textbook, 2012.

[9] V. Bruneau, K. Pankrashkin, and N. Popoff, Eigenvalue counting function for Robin Laplacians on conical domains, J. Geom. Anal. 28 (2018), 123-151.

[10] V. Bruneau and N. Popoff, On the negative spectrum of the Robin Laplacian in corner domains, Anal. PDE. 9 (2016), 1259-1283.

[11] J. Brüning, V. Geyler and K. Pankrashkin, Spectra of self-adjoint extensions and applications to solvable Schrödinger operators, Rev. Math. Phys. 20 (2008), 1-70.

[12] D. Daners, Robin boundary value problems on arbitrary domains, Trans. Amer. Math. Soc. 352 (2000), 4207-4236.

[13] V. A. Derkach, S. Hassi, M. M. Malamud and H. de Snoo, Boundary relations and their Weyl families, Trans. Amer. Math. Soc. 358 (2006), 5351-5400.

[14] V.A. Derkach and M. M. Malamud, Generalized resolvents and the boundary value problems for Hermitian operators with gaps, J. Funct. Anal. 95 (1991), 1-95.

[15] V.A. Derkach and M. M. Malamud, The extension theory of Hermitian operators and the moment problem, J. Math. Sci. 73 (1995), 141-242.

[16] P. Exner and P. Šba, A simple model of thin-film point contact in two and three dimensions, Czechoslovak J. Phys. B 38 (1988), 1095-1110.

[17] R. L. Frank and A. Laptev, Spectral inequalities for Schrödinger operators with surface potentials, in: Spectral Theory of Differential Operators, Amer. Math. Soc. Transl. Ser. 2, 225 (2008), pp. 91-102.

[18] F. Gesztesy and M. Mitrea, Nonlocal Robin Laplacians and some remarks on a paper by Filonov on eigenvalue inequalities, J. Differential Equations 247 (2009), 2871-2896.

[19] V.I. Gorbachuk and M. L. Gorbachuk, Boundary Value Problems for Operator Differential Equations, Kluwer Academic Publ., Dordrecht, 1991.

[20] G. Grubb, Distributions and Operators, Graduate Texts in Mathematics 252, Springer, New York, 2009. 
[21] G. Grubb, Spectral asymptotics for Robin problems with a discontinuous coefficient, J. Spectr. Theory 1 (2011), 155-177.

[22] D. D. Haroske and H. Triebel, Distribution, Sobolev Spaces, Elliptic Equations, EMS Textbooks in Mathematics, Zürich, 2008.

[23] B. Helffer and A. Kachmar, Eigenvalues for the Robin Laplacian in domains with variable curvature, Trans. Amer. Math. Soc. 369 (2017), 3253-3287.

[24] B. Helffer A. Kachmar, and N. Raymond, Tunneling for the Robin Laplacian in smooth planar domains, Commun. Contemp. Math. 19 (2017), 1650030, 38 pp.

[25] B. Helffer and K. Pankrashkin, Tunneling between corners for Robin Laplacians, J. London Math. Soc. 91 (2015), 225-248.

[26] T. Kato, Perturbation Theory for Linear Operators, Classics in Mathematics, SpringerVerlag, Berlin, 1995.

[27] H. Kovařík and A. Laptev, Hardy inequalities for Robin Laplacians, J. Funct. Anal. 262 (2012), 4972-4985.

[28] M. Levitin and L. Parnovski, On the principal eigenvalue of a Robin problem with a large parameter, Math. Nachr. 281 (2008), 272-281.

[29] V. Lotoreichik and J. Rohleder, Schatten-von Neumann estimates for resolvent differences of Robin Laplacians on a half-space, in: Spectral Theory, Mathematical System Theory, Evolution Equations, Differential and Difference Equations, Oper. Theory Adv. Appl. 221, Birkhäuser, Basel, 2012, pp. 453-468.

[30] M. Marletta and G. Rozenblum, A Laplace operator with boundary conditions singular at one point, J. Phys. A 42 (2009), 125204 (11pp).

[31] S. A. Nazarov and N. Popoff, Self-adjoint and skew-symmetric extensions of the Laplacian with singular Robin boundary condition, arXiv:1711.09654

[32] K. Pankrashkin and N. Popoff, Mean curvature bounds and eigenvalues of Robin Laplacians, Calc. Var. PDE 54 (2015), 1947-1961.

[33] K. Pankrashkin and N. Popoff, An effective Hamiltonian for the eigenvalue asymptotics of the Robin Laplacian with a large parameter, J. Math. Pures Appl. 106 (2016), 615-650.

[34] J. Rohleder, Strict inequality of Robin eigenvalues for elliptic differential operators on Lipschitz domains, J. Math. Anal. Appl. 418 (2014), 978-984.

[35] E. M. Stein, Singular Integrals and Differentiability Properties of Functions, Princeton University Press, 1970.

[36] G. Teschl, Mathematical Methods in Quantum Mechanics. With Applications to Schrödinger Operators, American Mathematical Society, Providence, 2009.

Institut für Angewandte Mathematik, Technische Universität Graz, Steyrergasse 30, 8010 Graz, Austria

E-mail address: behrndt@tugraz.at

$U R L:$ http://www.math.tugraz.at/ behrndt/

Institut für Angewandte Mathematik, Technische Universität Graz, Steyrergasse 30, 8010 Graz, Austria

E-mail address: schlosser@tugraz.at 